\title{
Numerical Simulation Analysis Based on the Study of the Slag discharge Mechanism of the Triangular-shaped Drill Pipe
}

\author{
Tang Dayong ${ }^{1}$, * , Tang $\mathrm{Yi}^{3}$, Duan Zhengyong ${ }^{1}{ }^{, 2}$, Lei wannian ${ }^{4}$, He Xiaolong ${ }^{1,2}$, Zhang Fubing ${ }^{1,2}$ \\ ${ }^{1}$ The School of Mechanical and Electrical Engineering, Chongqing University of Arts and Sciences \\ ${ }^{2}$ The Institute of Intelligent Equipment Manufacturing and Information Technology, Chongqing University of Art and Sciences, \\ Chongqing Yongchuan, 402160, China \\ ${ }^{3}$ School of Mechanical and Electrical Engineering, Emei Campus, Southwest Jiaotong University, Emei, Sichuan, 614202, China \\ ${ }^{4}$ Chongqing Research Institute Co., Ltd, China Coal Technology Engineering Group, Chongqing 400039, China
}

\begin{abstract}
According to the characteristics of high efficiency and high hole forming rate of the triangularshaped drill pipe in the process of drilling in soft outburst coal seam,the slag discharge model of annular gap between the triangular-shaped drill pipe and hole wall is established by using the basic theory of computational fluid dynamics. Using the finite volume element method, the flow field characteristics of the annular flow area between drill pipe and hole wall are analyzed.Through the numerical simulation analysis, the velocity fluctuation effect of the annular flow field of the slag removal model is put forward, that is, the velocity fluctuation zone is formed between the sides of the arc edge of the triangular-shaped drill pipe, which causes the precipitated coal cinder to rise under the vortex action of the flow field, and is quickly discharged out of the hole under the coordination of the axial velocity, which effectively improves the slag removal efficiency and pore forming rate of the drill pipe.Finally, the comparative test of the triangularshaped drill pipe and wide-blade spiral drill pipe was carried out in the soft outburst coal seam of 31021 lower auxiliary roadway in a mine. The drilling depth was increased by $19.4 \%$, the hole forming rate was increased by $17.4 \%$, and the drilling efficiency was increased by $26.4 \%$. There was no drill drop and broken phenomenon.
\end{abstract}

\section{Introduction}

With the increase of coal mining depth, the number of soft outburst mines is increasing year by year, and most of them have outburst risk due to soft coal seam, poor permeability and large gas content, which brings great difficulty to coal mine gas control ${ }^{[1-2]}$. At present, the most effective and successful method of gas control in domestic coal mines is still the coal seam drilling pre drainage technology. According to the mine gas control scheme and drilling design requirements, the full hydraulic drilling rig is used to drill into the coal seam, and then the drainage pipe is inserted into the hole to drain the gas out of the coal seam, so as to reduce the coal seam gas pressure and reduce the harm caused by coal seam mining ${ }^{[3-6]}$. However, due to the rich gas in the soft outburst coal seam and the influence of in-situ stress, the hole wall is extremely unstable.Under the action of drill pipe rotation and hydraulic scouring, it is prone to accidents such as the hole wall collapse, holding drill, breaking drill and dropping drill accidents, which seriously restricts the construction of gas drainage hole in the soft outburst mine ${ }^{[7]}$.
In recent years, triangular-shaped drill pipe or spiral drill pipe is generally used in the construction of gas drainage hole in soft outburst mine, especially the triangular-shaped drill pipe is widely used. In the process of drilling, triangular-shaped drill pipe has stronger slag discharge ability and efficiency than smooth round drill pipe and spiral drill pipe, which can quickly form holes in soft outburst coal seam. Hou Hong et al. ${ }^{[8-9]}$ think that the triangular-shaped drill pipe has larger slag discharge space than the smooth drill pipe, and the arc edge of the drill pipe can cut and roll the coal body, so that the coal cinder can be changed more finely, and the drill pipe has stronger slag discharge ability under the action of air pressure; Wang Yonglong et al. ${ }^{[10]}$ conducted numerical simulation research on the slag discharge principle of the triangular-shaped drill pipe, and compared it with the round drill pipe, and qualitatively analyzed the reasons for the rapid slag removal of triangular-shaped drill pipe; Xue Fei ${ }^{[11]}$ established the load model of triangularshaped drill pipe and deduced the analytical calculation method of drill pipe torque.

With the more and more complex geological conditions, the problem of long drilling in soft outburst coal seam will still be a technical problem in the

${ }^{*}$ Corresponding author: e-mail: tdylq@126.com 
construction of mine gas drainage hole for a long time. Therefore, it is necessary to deeply study the slag discharge mechanism of the triangular-shaped drill pipe, establish the physical model of slag discharge between drill pipe and hole wall, and obtain the key factors affecting the slag discharge of drill pipe. By using the numerical simulation method to analyze the drill pipe slag discharge, a set of theoretical method of drill pipe design is established, which provides technical support for the subsequent development of new drill pipe and brings drilling tools with excellent performance for coal mine gas control, which will surely produce greater economic and social benefits.

\section{Study on slag discharge mechanism of triangular-shaped drill pipe}

\subsection{Basic assumptions}

The cross-section of the triangular-shaped drill pipe is composed of three straight lines and three arc lines. When the drill pipe rotates at a high speed in the hole, the three arc edges and straight lines stir up the coal cinder in the hole, so that the coal cinder is suspended in the hole. Under the action of pressure air, the suspended coal cinders are discharged out of the hole. In the drilling process of drill pipe dry slag discharge with compressed air, the annulus area between drill pipe and hole wall can be regarded as gas-solid two-phase flow, so the drilling slag discharge model as shown in Fig.1 is established, and the following assumptions are made for the model ${ }^{[11-13]}$ :

1) The pressure gas can be regarded as ideal gas and should satisfy the equation of state of ideal gas;

2) The coal cinder are evenly distributed in the chip discharge annulus, and there is no phenomenon of heat transferring, mass transferring and velocity slipping between interphases.

3) The drill pipe axis is coaxial with the drilling axis;

4) The drilling speed is constant and the mass flow rate of coal cuttings is constant.

\subsection{Establishment of physical model}

In the axial section of the borehole, as shown in Fig. 1 (a), under the action of high-speed airflow, the coal cinder flows at high speed between the drill pipe and the hole wall. According to the Euler two fluid model theory, the governing equation of the annular flow field is established as ${ }^{[14-16]}$ :

Continuity equation:

$$
\begin{aligned}
& \text { Gas phase: } \frac{\partial}{\partial t}\left(\rho_{g} \alpha_{g}\right)+\nabla \bullet\left(\rho_{g} \alpha_{g} v_{g}\right)=0 \\
& \text { Solid phase: } \frac{\partial}{\partial t}\left(\rho_{k} \alpha_{k}\right)+\nabla \bullet\left(\rho_{k} \alpha_{k} v_{k}\right)=0
\end{aligned}
$$

The momentum conservation equation is as follows: Gas phase:

$$
\frac{\partial}{\partial \mathrm{t}}\left(\rho_{\mathrm{g}} \alpha_{g} \nu_{g}\right)+\nabla \bullet\left(\rho_{\mathrm{g}} \alpha_{g} \nu_{g} \nu_{g}\right)=-\alpha_{g} \nabla \bullet p_{g}+\nabla \cdot \tau_{g i j}-\beta\left(f_{g}-f_{k}\right)+\rho_{\mathrm{g}} \alpha_{g} g
$$

Solid phase:

$\frac{\partial}{\partial \mathrm{t}}\left(\rho_{k} \alpha_{k} v_{k}\right)+\nabla \bullet\left(\rho_{k} \alpha_{k} v_{k} v_{k}\right)=-\alpha_{k} \nabla \bullet p_{k}+\nabla \bullet \tau_{g i j}-\beta\left(f_{g}-f_{k}\right)+\rho_{k} \alpha_{k} g$

Where, $\rho_{g}$ is the density of the gas phase, $\rho_{k}$ is the density of the solid phase; $p_{g}$ is the pressure of the gas phase, $p_{k}$ is the pressure of the solid phase; $\alpha_{g}$ is the volume fraction of the gas phase, $\alpha_{k}$ is the volume fraction of the solid phase; $v_{g}$ is the velocity of the gas phase, $v_{k}$ is the velocity of the solid phase; $\nabla$ is the Hamiltonian operator, $\nabla=i \frac{\partial}{\partial x}+j \frac{\partial}{\partial y}+k \frac{\partial}{\partial z} ; \mathrm{g}$ is the acceleration of gravity; $\beta$ is the drag coefficient of the gas-solid two phases; $f_{g}$ and $f_{k}$ is the viscous stress produced on the surface of the micro element by the viscous action of the gas-solid two phases.

\subsection{Analysis of the slag discharge mechanism}

When high-speed airflow enters the annulus of the drill pipe from the drill pipe inner hole, the airflow velocity is high. The mass flow rate of bit coal drop remains unchanged or even increases, which leads to the decrease of axial velocity and precipitation. Nevertheless, from the borehole section, due to the abnormal profile structure of the drill pipe, the contour forms the minimum rotary diameter $\boldsymbol{D}_{\boldsymbol{l}}$ and the maximum rotary diameter $\boldsymbol{D}_{2}$ while rotating, as shown in Fig .1(b). In the cross-section, the coal cinder will be subjected to gravity, the force and friction force of the profile against the coal cinder, and the centrifugal force when the drill pipe rotates.On the upper side of the face (the left part of the center line of the section), the centrifugal force and the force of the drill pipe profile against the coal cinder are much greater than the gravity and friction force, and the resultant force will prevent coal cinders from continuing to sink, driving coal cinders upward and accelerating to the hole wall direction; On the lower side of the back (the right part of the center line of the section), the resultant force will make the coal cinder move downward and toward the hole wall.Most of coal cinders are suspended under the action of airflow, and a few of coal cinders precipitate at the bottom of the hole. When the arc edge of the drill pipe turns to the bottom, the deposited coal cinders will be again scraped by the edge surface and the arc edge, then become suspended. The mechanism of scraping and throwing up coal scraps by triangular-shaped drill pipe makes most of the coal scraps in the annular area of drill pipe in suspended motion for a long time. Under the action of high speed airflow, the coal cinder is continuously discharged out of the hole, which is the fundamental reason why the triangular-shaped drill pipe can quickly discharge slag 
and has high pore forming rate. The stress condition of coal cinders in the drilling section is shown in Fig. 1 (b).

The instantaneous acceleration of coal cinders moving towards the hole wall is as follows:

$$
a_{i}=\frac{F_{i}+\mathrm{N}-\mathrm{mg} \sin \theta}{m}
$$

The rising speed of coal cinders is as follows:

$$
V_{\text {up }}=V_{i} \sin \theta=\varpi \bullet r_{i} \sin \theta
$$

Where, $a_{i}$ is the instantaneous acceleration of the coal cinder, $F_{i}$ is the instantaneous centrifugal force on the coal cinder, its magnitude is: $F_{i}=m \frac{V_{i}^{2}}{r_{i}}=m \varpi^{2} r_{i}$,

$\boldsymbol{N}$ is the force of the drill pipe edge against the coal cinder, $\boldsymbol{M}$ is the mass of the coal cinder, $\theta$ is the angle between the position of the coal cinder and the horizontal direction, $\varpi$ is the angular velocity of the drill pipe, $r_{i}$ is the position of the coal cinder, $r_{i}=\left[D_{1} / 2, D_{2} / 2\right]$.
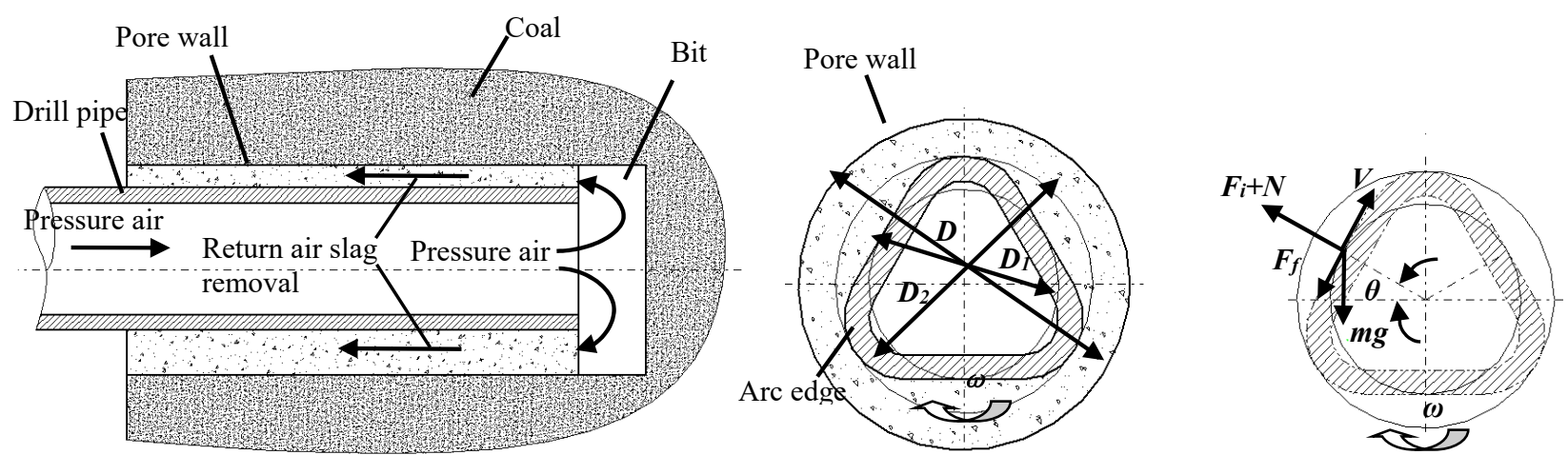

(a) Axial diagram of drilling

(b) Schematic diagram of stress and movement of drillingsection and coal cinders

Fig.1 Physical model of drilling slag discharge for the triangular-shaped drill pipe

\section{Establishment of geometric model and mesh generation}

\subsection{Geometric modeling}

In order to simplify the calculation, the length of the slag discharge model is $1000 \mathrm{~mm}$, the radius of arc edge is $\mathrm{R} 15$, the diameter of the drill pipe circumcircle is $73 \mathrm{~mm}$, and the diameter of the drill hole is $150 \mathrm{~mm}$. The slag discharge model is divided into two parts: drill pipe contour rotation and hole wall annular slag clearance. UG 3D modeling software is used to model the two parts of contour rotation and hole wall ring space gap, and it is assembled into a drill pipe slag discharge calculation model, which is shown in figure 2.

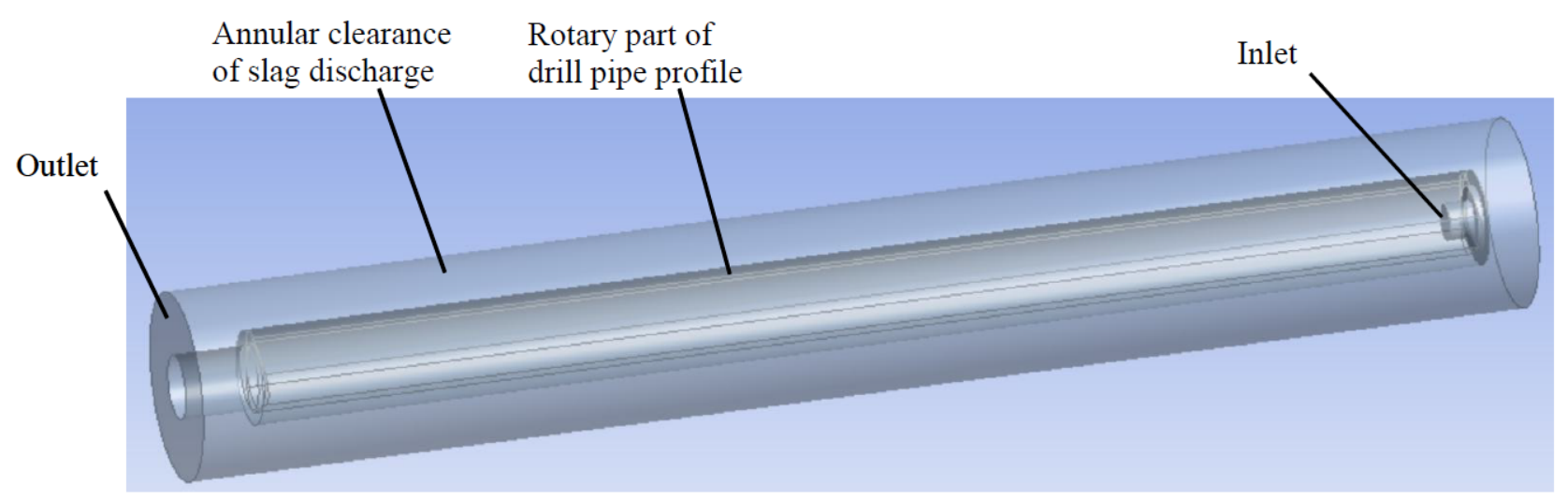

Fig.2 Analysis and calculation model of the triangular-shaped drill pipe

\subsection{Grid division of the model}

By using the geometric modeling module in the ANSYS Workbench software, the inlet and outlet, wall and interface are set up for the rotary part of the drill pipe contour and the annular clearance part of slag discharge.
In the meshing software, the boundary layer of the drill pipe contour wall will be set to control the meshing quality of the interface between the rotary part of the drill pipe contour and the annular clearance part of slag discharge.The grid division model is shown in Fig.3, with 5615186 cells and 1835142 nodes. 


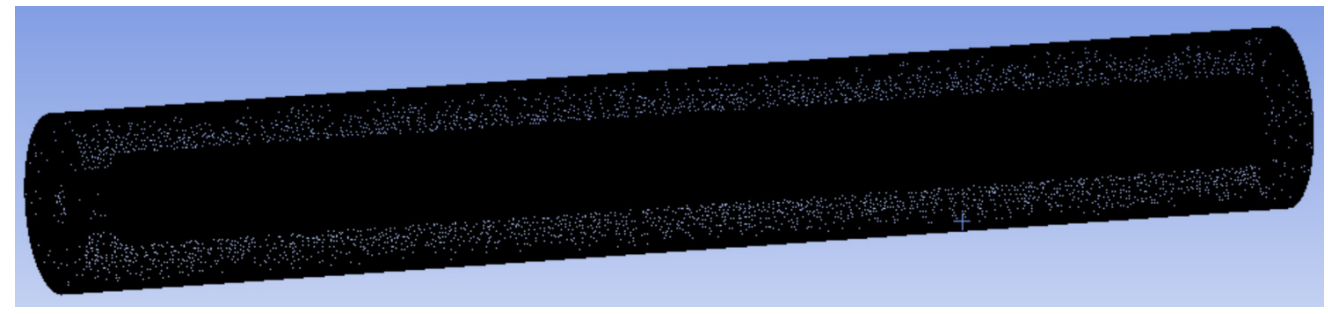

Fig.3 Grid division model of the triangular-shaped drill pipe

\section{Numerical simulation and result analysis of analytical model}

\subsection{Boundary conditions}

The Fluent module of ANSYS Workbench is used to simulate and analyze the calculation model.The boundary conditions of the model are as follows: the inlet speed is set to $38 \mathrm{~m} / \mathrm{s}$ (equivalent to $10 \mathrm{~m}^{3} / \mathrm{min}$ ), the outlet is set to free outflow, and the rotation speed of the drill pipe is set to $300 \mathrm{r} / \mathrm{min}$.

\subsection{Results analysis}

\subsubsection{Selection of observation surface and lines}

In order to accurately analyze the flow characteristics of annular clearance flow field of drill pipe and explore the slag discharge mechanism of triangular-shaped drill pipe, the axial cutting plane of slag discharge model, three groups of lines in the cutting plane (located on three cylindrical surfaces with different diameters, symmetrical up and down), the borehole section and lines in the section are selected as the flow field data observation surface and observation lines, as shown in Fig.4. The axial observation lines are taken as three groups of lines located in different diameter cylinders on the slag discharge model and parallel to the $\mathrm{Z}$ axis to observe the variation of axial velocity (slag discharge velocity) along the drilling depth, and the section observation surfaces are $Z=35 \mathrm{~mm}, Z=500 \mathrm{~mm}$ and $Z=1000 \mathrm{~mm}$ to observe the distribution of each surface velocity; The section observation line is taken from two groups of observation lines parallel to the edge plane (parallel to the edge plane and $3 \mathrm{~mm}$ apart ) and $\mathrm{Y}=0$ on the two sections of the $Z=500 \mathrm{~mm}, Z=1000 \mathrm{~mm}$ to see the change of axial velocity and tangential velocity on the section observation line.

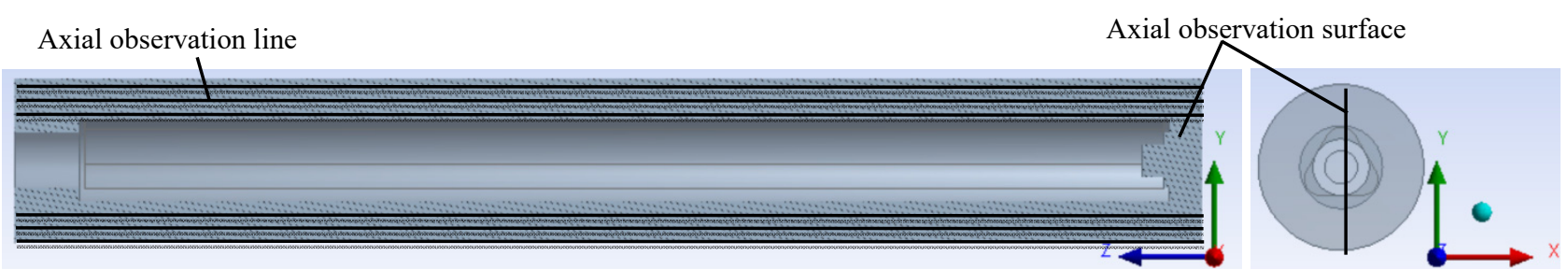

(a) Schematic illustration of axial observation surface and axial observation lines

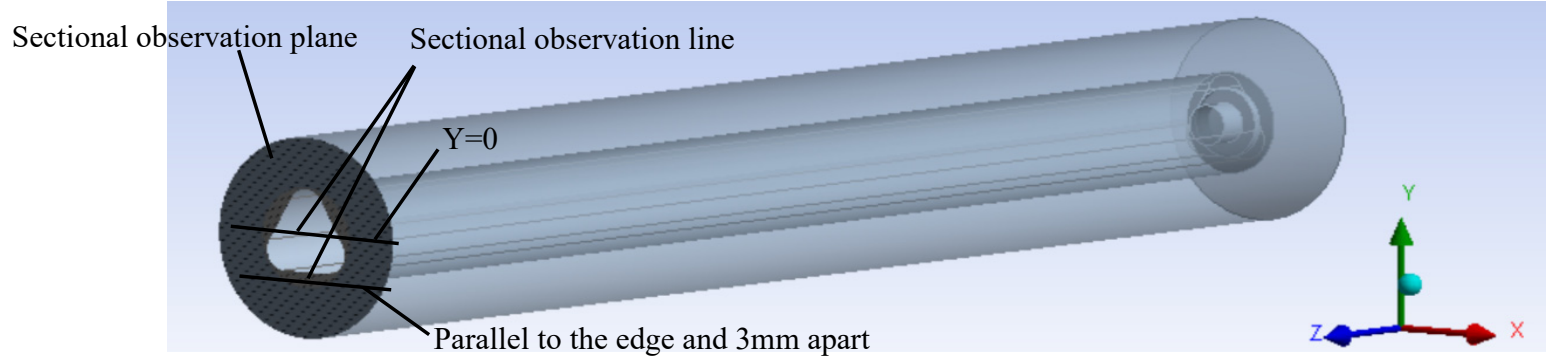

(b) Schematic illustration of the observation surface and the observation line of the section

Fig.4 Schematic diagram of observation surface and observation line position

\subsubsection{Distribution of axial velocity}

In the process of drilling, axial velocity(According to the modeling, the axial velocity is the z-direction velocity, the same below) plays a major role in drill pipe slag discharge, which directly discharges out of the hole. Therefore, the distribution of axial velocity in the annular flow field of drill pipe slag discharge can be analyzed by the data of the drill pipe axial observation surface, as shown in Fig.5. As can be seen from the axial velocity distribution vector figure, there is a large vortex in the flow field at the bottom of the hole, where the coal cinder is repeatedly cut and rolled to form relatively small pulverized coal, which is easy to discharge out of the hole. However, at the orifice, the axial velocity at the arc edge near the hole wall is greater than that at the outer plane of the drill pipe, indicating that edges of the triangular-shaped drill pipe have the effec of stirring the coal cinder, which is conducive to coal cinders 
discharging out of the hole.From the distribution curve of the axial velocity on the axial observation line (as shown in Fig.6(a)) and the distribution nephogram of the axial velocity on the section observation surface (as shown in Fig. 7 (a)), the maximum axial velocity is 17 $\mathrm{m} / \mathrm{s}$ at the place where the compressed air returns from the bottom of the hole, and it is distributed on the outer wall of the circumference of the hole. A negative pressure zone is formed near the arc edge of the drill pipe away from the rotation direction, and the minimum axial velocity is $-0.8 \mathrm{~m} / \mathrm{s}$.According to Fig. 6 (b)、Fig. 7 (b) and (c), with the coal cinder moving out of the hole, the "velocity fluctuation effect" is generated in the annular slag discharge space under the action of the arc edges of the triangular-shaped drill pipe.From the crosssection of the borehole, the annular slag discharge space is divided into three "velocity fluctuation zones" by arc edges. The axial velocity fluctuates in the range of $1 \sim 3.5 \mathrm{~m} / \mathrm{s}$, and the maximum velocity is $3.5 \mathrm{~m} / \mathrm{s}$ near the hole wall near the arc edge. The "velocity fluctuation effect" of the slagging space in the annular space of the triangular-shaped drill pipe makes the coal cinder in the hole always move, which greatly improves the slag discharging quantity and efficiency.

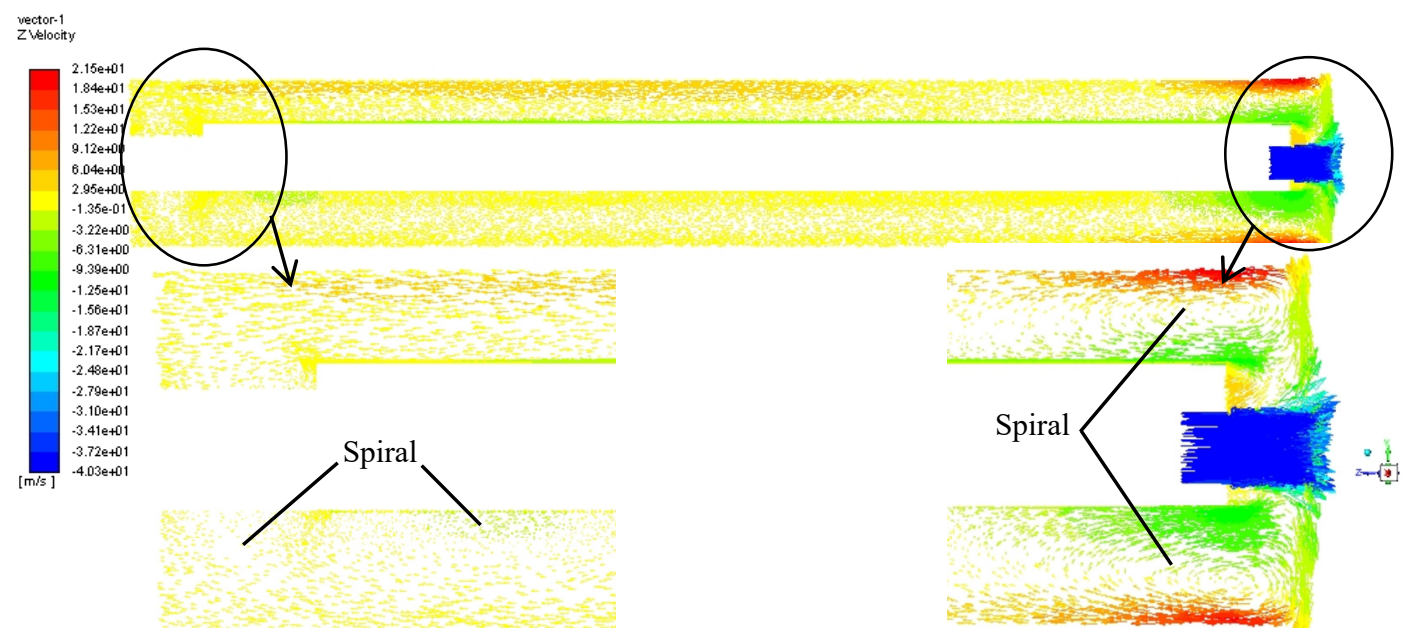

Fig.5 Axial velocity vector diagram of annular clearance flow field of the triangular-shaped drill pipe

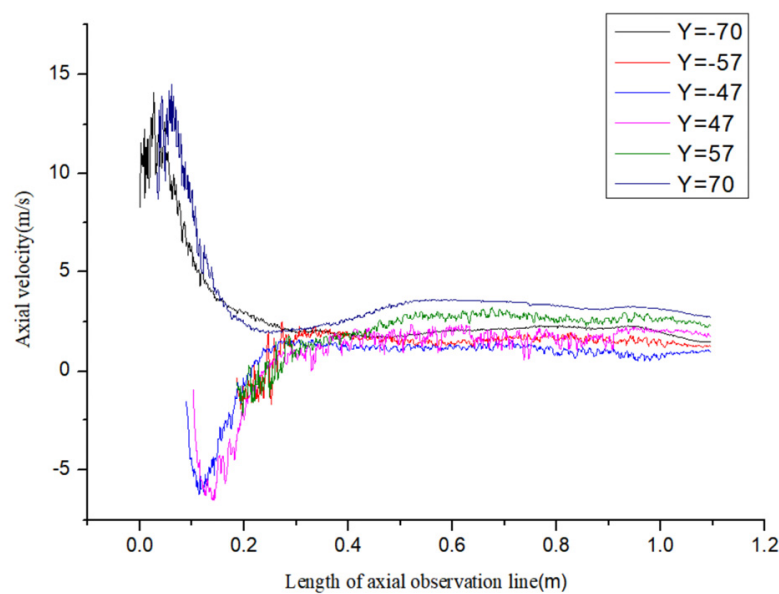

(a) Distribution of axial velocity on axial observation line

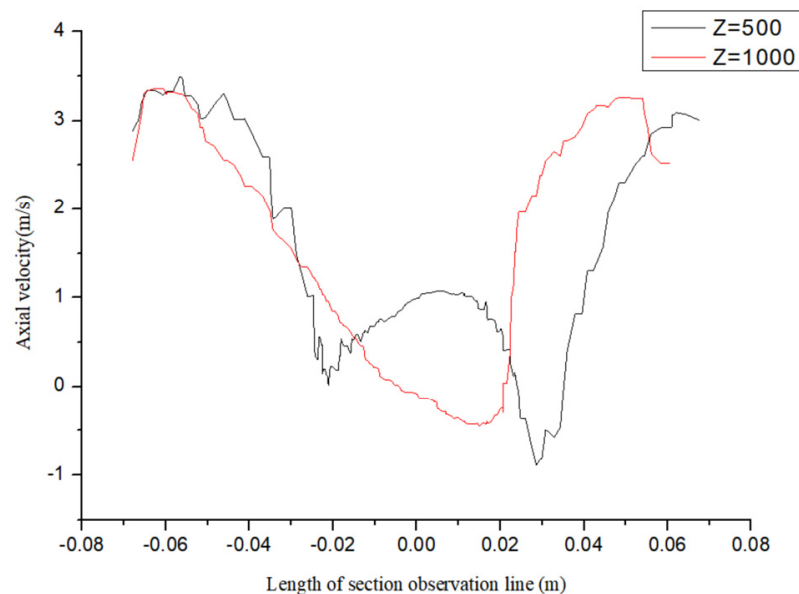

(b) Distribution of axial velocity on section observation line

Fig.6 Distribution of axial velocity 

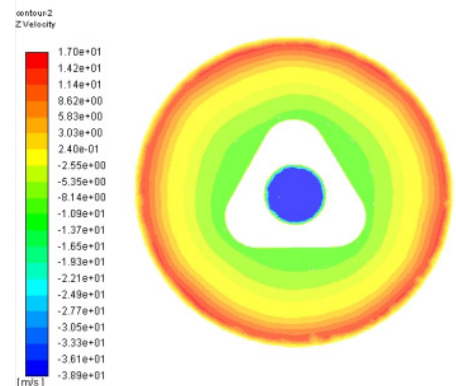

(a) Nephogram of the axial velocity at $\mathrm{z}=35 \mathrm{~mm}$
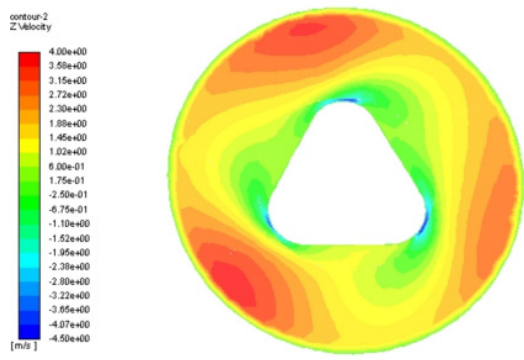

(b) Nephogram of the axial velocity at $\mathrm{z}=500 \mathrm{~mm}$

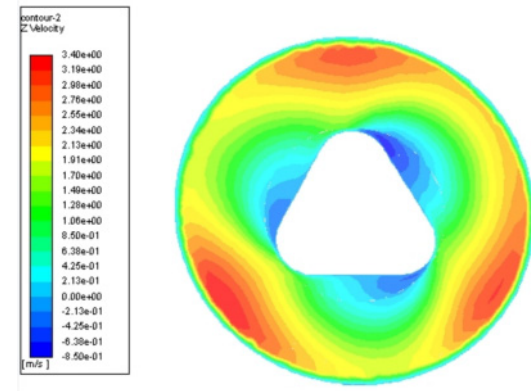

(c) Nephogram of the axial velocity at $\mathrm{z}=1000 \mathrm{~mm}$

Fig.7 Nephogram of axial velocity distribution of cross-section

\subsubsection{Distribution of tangential velocity}

During drilling, with the deepening of the borehole, the coal cinder in the hole will be continuously precipitated at the bottom of the hole, but the arc edges of the triangular-shaped drill pipe have the function of scraping the coal cinder precipitated in the hole, which makes the coal chips rise and discharge the coal cinder out of the hole under the action of axial velocity.The axial observation line shows that the tangential velocity fluctuates in a small range of about $1 \mathrm{~m} / \mathrm{s}$, but the closer the drill pipe edge line, the greater the velocity fluctuation, as shown in Fig .8(a).The maximum tangential velocity near arc edges of the triangularshaped drill pipe is about $0.5 \mathrm{~m} / \mathrm{s}$. With the increase of the radius, the tangential velocity decreases gradually and reaches the minimum at the hole wall. The range of 36.5 to $36.5 \mathrm{~mm}$ is the profile of the drill pipe , and the data in this section are invalid data(the same below), as shown in Fig. 8 (b).

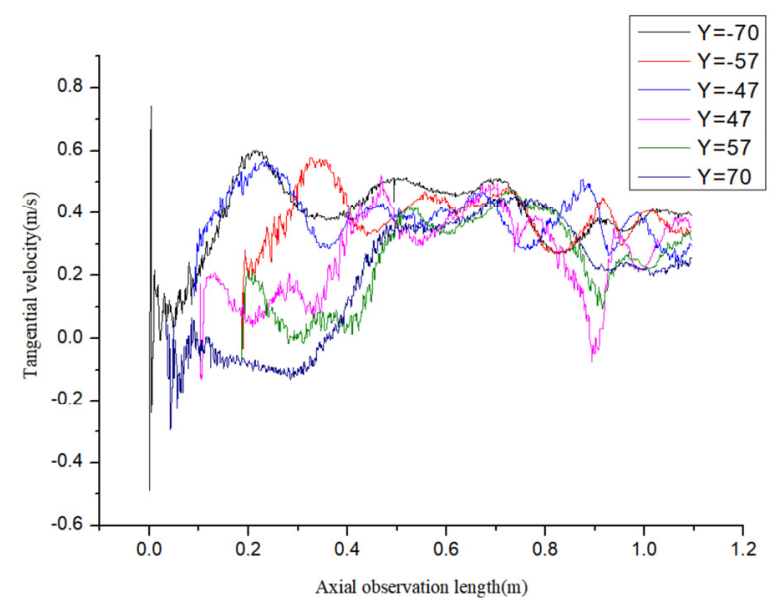

(a) Distribution of tangential velocity on axial observation line

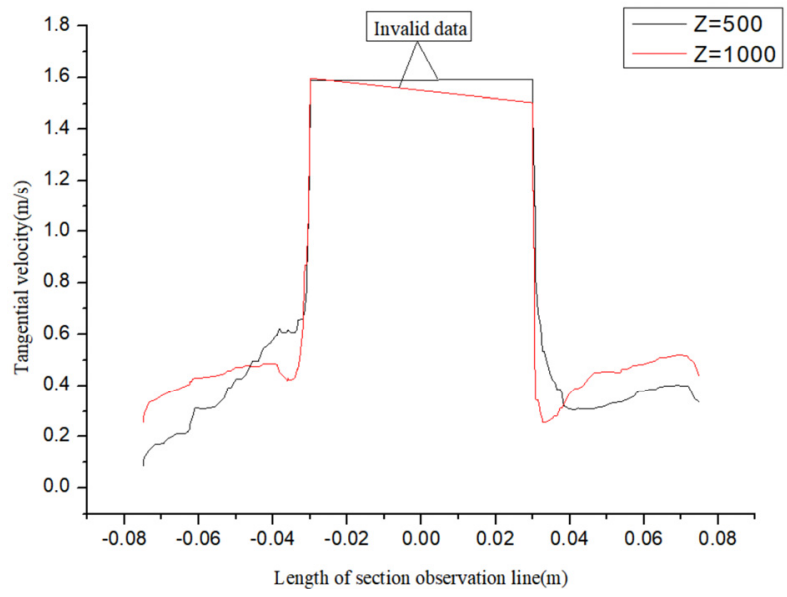

(b) Distribution of tangential velocity on observation lines of different sections

Fig.8 Distribution of tangential velocity

\subsubsection{Effect of borehole gradation combination on velocity}

\subsubsection{Effect of gradation combination on axial velocity}

The parameters of the drill pipe were kept constant, and the diameter of the model borehole was changed from $150 \mathrm{~mm}$ to $94 \mathrm{~mm}$. The distribution of axial velocity on the axial observation line was shown in Fig.9. As it can be seen from Fig.9 (a), with the decrease of borehole gradation, the axial velocity at the outlet increases by about $2 \mathrm{~m} / \mathrm{s}$, up to $4 \sim 6 \mathrm{~m} / \mathrm{s}$. The axial velocity at the bottom of the hole is as high as $27 \mathrm{~m} / \mathrm{s}$, which is $10 \mathrm{~m} / \mathrm{s}$ higher than the original gradation, and the ability of returning slag at the bottom of the hole is greatly improved. The axial velocity distribution on the observation lines of $Z=500 \mathrm{~mm}$ and $1000 \mathrm{~mm}$ sections is in the range of $3 \sim 7.3 \mathrm{~m} / \mathrm{s}$, and the velocity fluctuation effect near the edges is enhanced, as shown in Fig.9 (b) . 


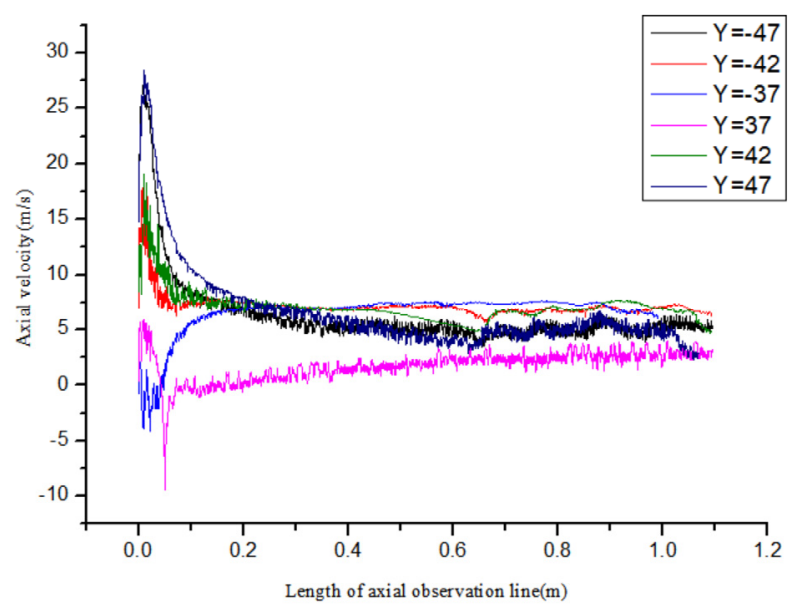

(a) Axial velocity distribution on axial observation line

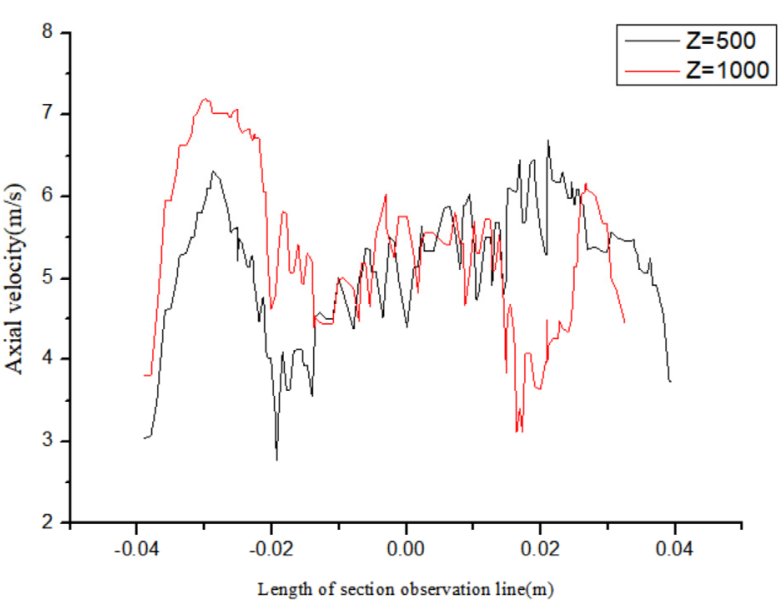

(b) Distribution of axial velocity on observation lines of different sections

Fig.9 Distribution of axial velocity after decreasing gradation

\subsubsection{Effect of gradation combination on tangential velocity}

As it can be seen from Fig.10 (a), with the decrease of borehole gradation, the fluctuation effect of the tangential velocity of annular clearance flow field of drill pipe is enhanced, especially near the arc edge of drill pipe, where the velocity changes significantly

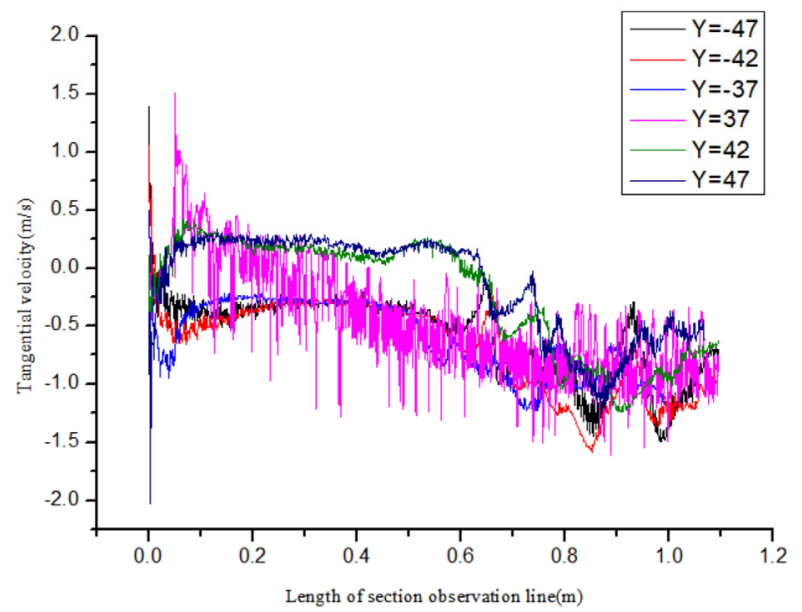

(a) Distribution of tangential velocity on axial observation line
( $Y=37 \mathrm{~mm}$ ), up to $3 \mathrm{~m} / \mathrm{s}$. While it can be seen from Fig.10 (b), the tangential velocity on the observation line of $\mathrm{Z}=$ $500 \mathrm{~mm}$ and $1000 \mathrm{~mm}$ cross-sections is almost reversed (negative number only represents the direction, the same below). The "velocity fluctuation effect" is enhanced. The coal cinder in the hole are difficult to precipitate under this effect, which has a good role in improving the efficiency of drill pipe slag discharge.

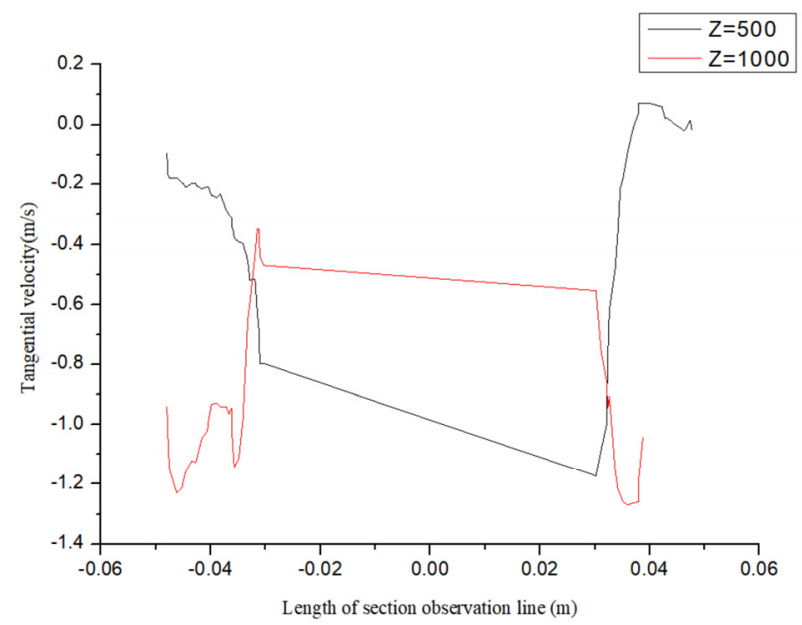

(b) Distribution of tangential velocity on observation lines of different sections

Fig.10 Distribution law of tangential velocity after decreasing gradation

\subsubsection{Effect of edge radius on velocity}

\subsubsection{Effect of edge radius on axial velocity}

Keeping the circumcircle diameter $\Phi 73$ of the drill pipe and the drilling diameter $\Phi 94$ invariant, the radius of the arc edge of drill pipe cross-section is reduced from R15 to R10 on the premise of sufficient strength of drill pipe connection thread.Then, the flow field in annular clearance space of drill pipe is numerically simulated,and the axial velocity distribution on the axial observation line and section observation line is shown in Fig.11.The axial velocity on the axial observation line and the flow field change little with the decrease of gradation, and the outlet velocity is still $4 \sim 6 \mathrm{~m} / \mathrm{s}$. But the axial velocity tends to be uniform on the $Z=500 \mathrm{~mm}$ and 1000 sections, and the velocity fluctuation near the arc edge ranges from $3.25 \mathrm{~m} / \mathrm{s}$ to $6.75 \mathrm{~m} / \mathrm{s}$. 


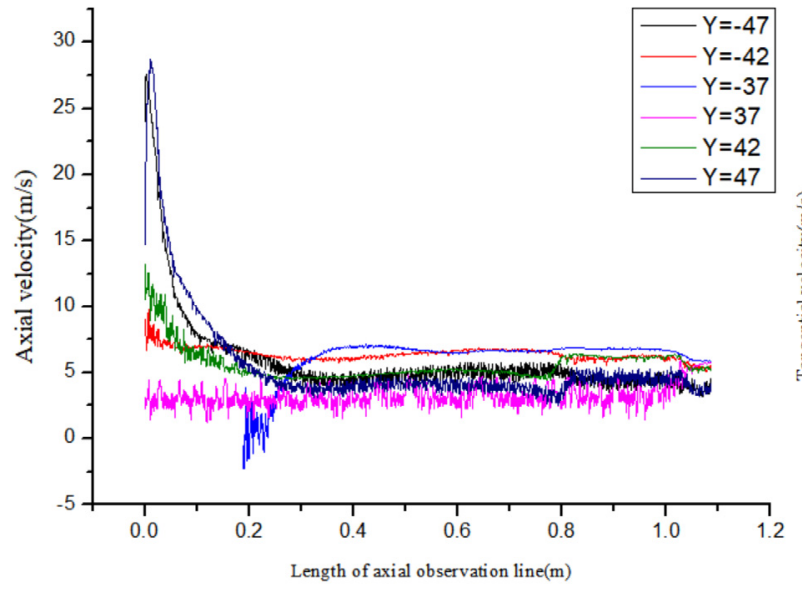

(a) Distribution of Axial velocity on axial observation line

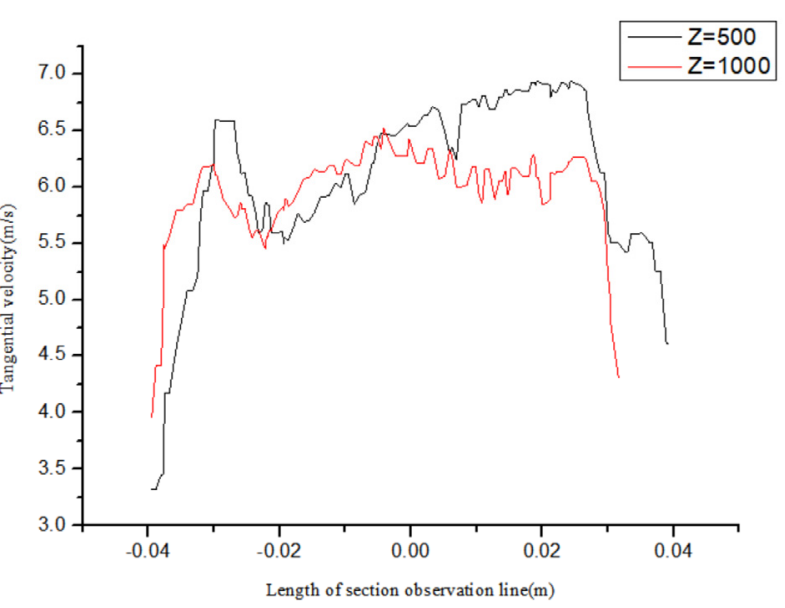

(b) Distribution of axial velocity on observation lines of different sections

Fig.11 Distribution of axial velocity after decreasing radius of the arc edge

\subsubsection{Effect of edge radius on tangential velocity}

As it can be seen from Fig.12 (a) that when the radius of the arc edge of the drill pipe decreases, the tangential velocity fluctuation effect on the nearest observation line $(\mathrm{Y}=37)$ from the drill pipe edge line is further enhanced, and a larger vortex is generated in the annular gap, which enhances the tangential velocity fluctuation in the flow field of the drill pipe slag clearance, so that the coal cinder in the hole is in a rotating state, and is quickly discharged out of the hole under the drive of the axial velocity. In Fig.12 (b), the tangential velocity is reversed on the observation lines of $\mathrm{Z}=500 \mathrm{~mm}$ and $1000 \mathrm{~mm}$, and the "velocity fluctuation effect" is further enhanced, which greatly improves the slag removal efficiency of drill pipe.

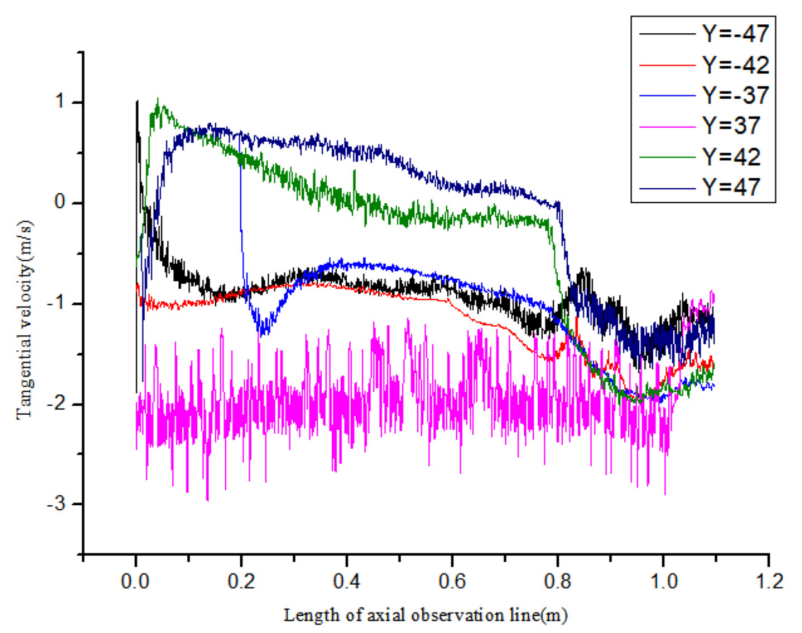

(a) Distribution of tangential velocity on axial observation line

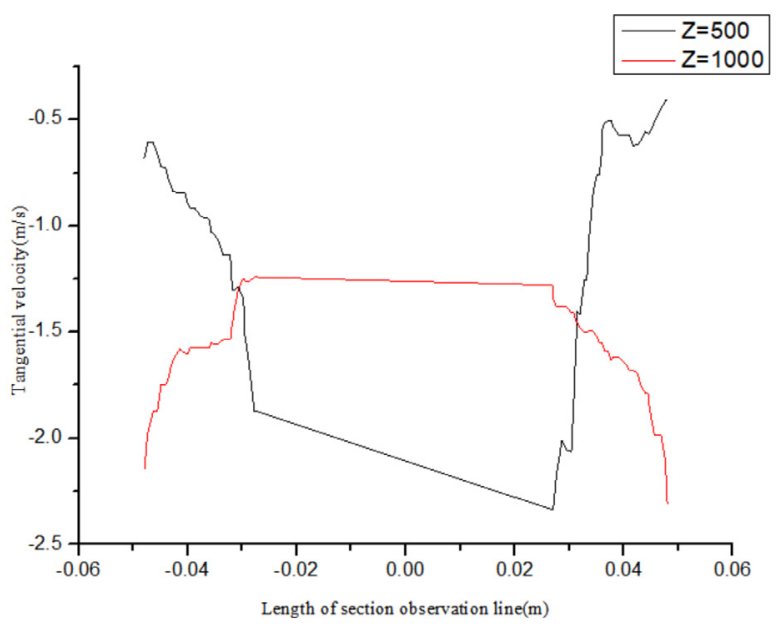

(b) Distribution of tangential velocity on observation lines of different sections

Fig.12 Distribution of tangential velocity after decreasing radius of the arc edge

\section{Test verification and comparison}

\subsection{Geological conditions of drilling construction}

The coefficient of sturdiness of coal seam in 31021 sub roadway of a mine is 0.3 , soft coal seam, $3 \sim 4 \mathrm{~m}$ thick, poor permeability, and the hole wall collapses easily during drilling.At present, ZYW-4000 drilling rig, $\Phi 63 / 73$ wide-blade spiral drill pipe and $\Phi 94$ drill bit are used for bedding drilling in soft coal seam. The designed drilling depth is $85 \mathrm{~m}$. In order to verify the rationality of the slag discharge structure design of the triangularshaped drill pipe, the triangular-shaped drill pipe with diameter of $\Phi 73$ was provided for the mine without changing the working condition of the drill rig. The edge 
radius of drill pipe is R10 (see Fig.13 for outline), and the matching drill bit is $\Phi 94$.

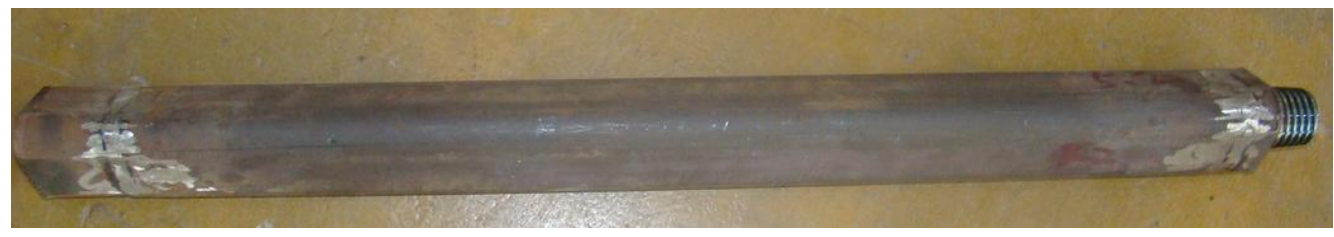

Fig.13 Outline structure diagram of the triangular-shaped drill pipe

\subsection{Analysis of test results}

The $\Phi 63 / 73$ wide-blade spiral drill pipe was used to construct 40 holes along the coal seam in 31021 working face of the mine.The average drilling depth is $85.6 \mathrm{~m}$, which lasts for 32 days, the cumulative footage is $3513.5 \mathrm{~m}$, and the hole forming rate is $75 \%$. The application of $\Phi 73$ triangular-shaped drill pipe in the same working condition has 53 holes along the coal seam.The average drilling depth is $102.2 \mathrm{~m}$ and the total footage is $5416 \mathrm{~m}$ in 39 days. The hole forming rate was $92.4 \%$.

The average hole depth is increased by $19.4 \%$, and the daily drilling efficiency is increased by $26.4 \%$, and the drilling test data is shown in Fig.14.

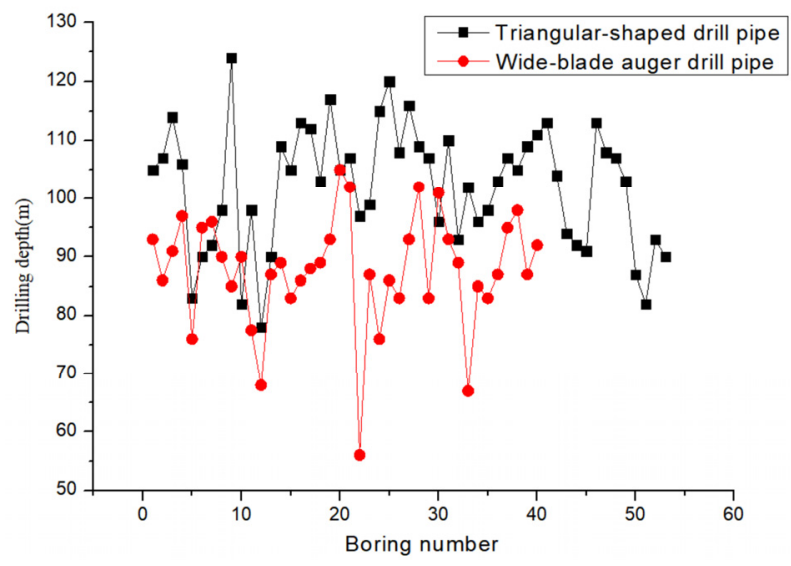

Fig.14 The results of borehole test

\section{Conclusion}

1) According to the basic theory of fluid dynamics and the numerical calculation method, this paper establishes the corresponding analysis model for the slag discharge structure of the triangular-shaped drill pipe, and points out that there is "velocity fluctuation effect" in the annular slag discharge space of the triangular-shaped drill pipe, which is the fundamental reason for the large amount of slag discharge and high drilling efficiency of the triangular-shaped drill pipe.

2) Under the same boundary conditions, it was simulated and analyzed for the slag discharge flow field in the annulus space of the drill pipe by changing the gradation combination and the radius of the cross-section arc edges of triangular-shaped drill pipe, and a design method of triangular-shaped drill pipe for reference is proposed.
3) According to requirements of the mine, a triangular-shaped drill pipe with circumscribed circle of $\Phi 73$ is designed.Using the existing drilling equipment, the triangular-shaped drill pipe and the original $\Phi 63 / 73$ wide-blade spiral drill pipe were drilled test under the same working conditions.Compared with wide-blade spiral drill pipe,the hole forming rate of triangularshaped drill pipe increased by $17.4 \%$, the drilling efficiency increased by $26.4 \%$, and the hole forming rate reached $76.3 \%$.In the process of drilling test, there are no drill pipe fracture, sticking, holding and other problems.

\section{Acknowledgments:}

Chongqing Banan District independent innovation and technology project (bnstc,202007); Chongqing Yongchuan District common key technology innovation key project (ycstc,2020ab); Chongqing University of Arts and Sciences talent introduction project (2017RJD17).

\section{Reference}

1. Zhang Mingjie, Yang Shuo. Mechanical mechanism of suction sticking in auger drill pipe drilling in soft coal seam $[\mathrm{J}]$. Coal geology and exploration,2015,43(05):121-124.

2. Gao Kui, Liu Zegong, Liu Jian, Deng Dongsheng, Kang Ya, Huang Kaifeng. Physical and mechanical properties of structural soft coal and its effect on coal and gas outburst [J]. China Safety Science Journal,2013,23(02):129-133.

3. Zhang Ying. Instability Mechanism Analysis and Reinforcement Technology of Gas Drainage Borehole in Soft and Broken Coal Seam [J]. Coal Science and Technology,2018,46(07):178-183.

4. GUO Chunsheng, XU Chao, GE Yong, GENG Dewang, HU Siyi, GONG Ke. Optimization of drainage time of floor through bed drilling in soft and low permeability coal seam in Yangquan mining area $[\mathrm{J}]$. Journal of $\mathrm{Xi}$ 'an University of Science and Technology,2019,39(05):875-881.

5. Li Hong, Hao Guangsheng. Deep hole drilling technology for gas drainage in soft coal seam of high outburst mine [J]. Journal of Heilongjiang University of Technology,2019,29(06):642-647.

6. Zhang Xuebo, Ji Zhongchao, Gao Jianliang. Numerical analysis of influencing factors of effective drainage radius of bedding borehole [J]. 
Journal of Safety and Environment, 2013,13(02):157-160.

7. Tang Dayong. Study on the special-shaped drill pipe and its construction technology in soft outburst coal seam [J]. Coal Mine Machinery,2011,32(04):220222.

8. Hou Hong, Ling Biacan, Luo Wei, Jin Xin, Wang Li, Liu Fayi. Research on drilling technology of triangular drill pipe in soft outburst coal seam [J]. Coal Science and Technology, 2012,40(08):67$70+74$.

9. Ling Biacan, Dai Shixin, Cao Chengping, Cao Pingxiao. Application of three-edge drill pipe in bed gas drainage drilling in medium and soft coal seam [J]. Coal Science and Technology,2009,37(10):44$46+111$.

10. Xue Fei. Research on calculation model of powder loading torque of triangular drill pipe [J]. China Science and technology of work safety, 2017,13 (02): 164-170.

11. Wang Yonglong, Liu Chun, sun Yuning, song Weibin, Wang Zhenfeng. Numerical simulation of slag removal principle of edge drill pipe in gas drainage hole $[\mathrm{J}]$. Journal of safety and environment, 2015,15 (04): 89-93.

12. Xiao Guoyi, Zhao Xiangyang, Meng Yingfeng, Zhu Huashu, Lian Zhanghua, Li Gao. Study on multiphase flow law at key points of rock carrying in gas drilling $[\mathrm{J}]$. Petroleum drilling and production technology, 2011,33 (01): 16-18+22.

13. Wang Qi. CFD model study and experimental verification of gas-solid fluidized bed two-phase flow [D]. Huazhong University of science and technology, 2012.

14. Chao Donghai, Guo Xueyan. CFD simulation of two-phase flow in large particle gas-solid fluidized bed [J]. Journal of Shanghai University of technology, 2010,32 (04): 333-339 + 358 .

15. Zhang Long lie. Mechanism of pneumatic chip removal in drill pipe inner hole of gas drainage hole in soft outburst coal seam [D]. Henan University of technology. 2015.

16. Yuan Zhulin. Gas solid two phase flow and numerical simulation [M]. Nanjing: Southeast University Press, 2013. 